\title{
Clinical application value of intraoperative transesophageal echocardiography in pediatric cardiac valvular disease
}

Jingjing Qian

Zhejiang University School of Medicine

Jingjing Ye ( $\square$ yejingjing578@126.com )

Zhejiang University School of Medicine

Jin Yu

Zhejiang University School of Medicine

Xiuzhen Yang

Zhejiang University School of Medicine

Lei Zhao

Zhejiang University School of Medicine

Research article

Keywords: Transesophageal echocardiography; Heart valve disease; Children

Posted Date: September 8th, 2019

DOI: https://doi.org/10.21203/rs.2.14170/v1

License: (9) This work is licensed under a Creative Commons Attribution 4.0 International License.

Read Full License 


\section{Abstract}

Background: To explore the value of intraoperative transesophageal echocardiography (TEE) in the surgical treatment of pediatric cardiac valvular disease.

Methods: Transthoracic echocardiography (TTE) was performed in 64 children with cardiac valvular disease, of which 49 patients underwent TEE before and after cardiopulmonary bypass.

Results: The coincidence rate of preoperative TEE with the surgical diagnosis on valvular lesions was $100 \%$, higher than that of TTE (78\%). TEE supplemented or corrected 10 cases of TTE valvular lesion diagnosis, improving the accuracy of valvular disease diagnosis. Besides, TEE can effectively evaluate the surgical results.

Conclusions: Preoperative TEE can accurately diagnose valvular disease in children, which helps to develop a surgical plan. Postoperative TEE can find the remaining problems in time and improve the success rate of surgery.

\section{Background}

Transesophageal echocardiography (TEE) examination has been widely used in coronary heart disease (CHD) and valvular disease surgery[1-5], showing important value for making treatment decisions. Before the advent of pediatric-specific TEE probes, we used transthoracic echocardiography (TTE) for children with heart disease. Compared with TTE, TEE has better sound-transparent conditions, especially for older children, obese children, and children with significant lung interference.

How to better use TEE to monitor the valve during the perioperative period is of great significance to improve the success rate of surgery and reduce complications. This study performed a TEE examination on patients and compared it with TTE to evaluate the value of TEE in the surgical treatment of children with cardiac valvular disease.

\section{Methods}

The present study was approved by the Ethics Committee of Department of ultrasound, Children's Hospital, Zhejiang University School of Medicine, Hangzhou, China. Written informed consent was obtained from all participants.

\section{Patients}

From January 2017 to March 2019, 64 children with valvular heart disease were enrolled, including 36 males and 28 females, aged from 1 month to 160 months, with an average of ( $40.3 \pm 41.1)$ months. There were 36 cases of common atrioventricular canal with valvular disease, 9 cases of Ebstein's anomaly, 8 cases of ventricular septal defect, atrial septal defect and patent ductus arteriosus combined with valvular lesion, 1 case of coarctation of aortic arch with mitral valve disease, 1 case of pulmonary 
stenosis with tricuspid valve disease, 2 cases of mitral stenosis with insufficiency, 1 case of mitral valve prolapse with cleft, 2 cases of two lobe malformation of aortic valve, 1 case of aortic-left ventricular canal with aortic insufficiency, and 3 cases of valvular disease caused by infective endocarditis (Figure $1-10)$.

Transthoracic echocardiography (TTE) was performed in 64 children with cardiac valvular disease, of which 49 patients underwent transesophageal echocardiography (TEE) before and after cardiopulmonary bypass.

Inclusion criteria: patients with congenital or secondary heart valve disease; children with calm coordination, and clear ultrasound images can thus be obtained; patients with at least two times of transthoracic heart Doppler ultrasound; preoperative, intraoperative, and postoperative ultrasound examinations were completed by a doctor with more than 13 years of experience; cardiac function ejection fraction (EF) $\geq 55 \%$; hospitalized patients with complete medical records; patients who can actively cooperate with the treatment and follow-up.

Exclusion criteria: patients with valvular regurgitation after heart surgery; patients with unexplained valvular regurgitation; patients who had not been diagnosed by surgical treatment; patients combined with fever, pneumonia (gas would affect the clarity of transthoracic ultrasound images when coughing), chest fluid, and pericardial effusion; cardiac function $\mathrm{EF}<55 \%$; patients without postoperative follow-up data.

Methods

TTE was performed on 64 children undergoing surgical treatment with a Philips iE33 color ultrasound system, S8-3 probe, S5-1 probe, GE VividE9 color Doppler ultrasound, M5S probe, and 6S probe. During the examination, the child was placed in the supine position or the left lateral position. The uncooperative person was sedated with $10 \%$ chloral hydrate to fully expose the chest. The heart anatomy was routinely scanned to determine whether the heart was deformed, with the valve carefully observed.

During the operation, the Philips iE33 ultrasound system was applied. The S7-3t and S8-3t were inserted through the esophageal probe, and the area associated with heart disease in 49 patients with general anesthesia was examined and compared with preoperative TTE results. The examination information was communicated to the cardiac surgeon in time and compared with the intraoperative findings. After the operation, the heart was re-explored and TEE was used to evaluate the effect of the operation.

\section{Results}

Comparison of preoperative TTE and TEE diagnosis results

There was no significant difference in baseline data between patients undergoing TTE and TEE $(P>0.05)$ (Table 1). The coincidence rate of TTE and surgical diagnosis of 64 children was $78 \%$ (50/64) (Table 2), 
of which 49 patients receiving preoperative and postoperative TEE obtained satisfactory two-dimensional and color Doppler sonograms. Especially for valvular lesions, TEE had more advantages than TTE, clearly showing the shape, echo, opening, and closing of the leaflets, fissures, as well as other malformations in the heart, which were basically consistent with the findings during the operation. Ten patients (Table 3) were diagnosed or corrected by preoperative TEE examination, and the coincidence rate with the surgical diagnosis was $100 \%(49 / 49)$.

Accuracy of the diagnosis of the valvular disease improved by TEE

TEE made a clear etiological diagnosis of valvular lesions diagnosed by preoperative TTE, including 10 cases of supplemental or modified diagnosis, consistent with surgical findings (Table 4).

Postoperative TEE assessment of surgical outcomes

Immediately after the operation, the TEE was evaluated, and 3 cases were found to have significant residual problems. The residual septal flow was greater than $2 \mathrm{~mm}$, and the flow velocity was greater than $3 \mathrm{~m} / \mathrm{s}$ in two cases; the valve was moderate insufficiency in one case. As the TEE was found timely, it was handled accordingly and the results were satisfactory.

The success rate of 49 cases of TEE probe insertion into the esophagus was $100 \%$. All children were able to tolerate TEE and no adverse complications occurred. For children with cardiopulmonary bypass, ventilatory guidance in the left heart system was performed under the observation of TEE before the end of cardiopulmonary bypass, and air embolism complications did not occur in all children.

\section{Discussion}

The most common cause of valvular disease in children is congenital heart disease, followed by infective endocarditis and rheumatic valvular disease. In this group of 64 children, 61 cases were the congenital valvular disease, of which 36 cases (56.25\%) were common atrioventricular canal with valvular disease, and 3 cases were caused by infective endocarditis.

The clinical application of TEE in pediatrics has greatly overcome the limitations of TTE and has become an important tool for the diagnosis of cardiac valvular disease in children.

TEE has an advantage in displaying the details of valvular lesions. In this group of patients, the common atrioventricular canal combined with left and right atrioventricular valve lesions, preoperative TEE can clearly show the development of chordae, papillary muscles, and valve leaflets, the degree of regurgitation, as well as small leaflets that are difficult to find by TTE. Postoperative TEE can accurately assess valve regurgitation and residual shunt at the atrioventricular septal repair. For the Ebstein's anomaly, the diagnostic accuracy of TTE is already high. On the basis of TTE, TEE can more clearly show the development of the downward or posterior flap, and accurately measure the distance of the downward movement. TEE is also superior to TTE in the presentation of the mitral, tricuspid papillary muscles and chordae, displaying the abnormally developed papillary muscles and rupture of chordae 
tendinca. TEE also accurately displays the number of aortic valve leaflets and the shape of the valve. For a child with aortic-left ventricular anal and aortic valve moderate insufficiency, TEE more clearly distinguishes between the aorta-left ventricle and the incomplete flap. For children with infective endocarditis, TEE can accurately display the size, number, and attachment of neoplasms, as well as the situation of leaflets [6-9]. In a case of the left coronary artery-right atrium with fistula, right atrium and aortic valve have neoplasms, and TEE is consistent with the surgical findings.

TEE can also detect post-operative problems in time and avoid the risk of reopening the chest. It can provide accurate and reliable information to the surgeon before and after surgery, which enhances the surgeon's confidence and effectively improves the success rate of the operation.

There were no complications in this group of patients receiving TEE. The probes designed for infants are softer and can be used for newborns. In addition, professional cardiac ultrasound doctors are required to operate, with correct and gentle manipulation when inserting and removing the probe. For patients with difficulty in insertion, the doctors use a laryngoscope. During the extracorporeal circulation, the TEE probe is frozen and left in the stomach, reducing the number of probes inserted and removed, which can reduce or even avoid the complications of TEE.

Real-time three-dimensional transesophageal echocardiography (RT-3D-TEE) can be acquired in real-time and imaged rapidly, displaying the three-dimensional structure of the heart in multi-directional visions and enabling the accurate analysis of valve anatomy, which is a new method to evaluate heart valve disease[4, 10]. In children, due to weight limitations, it is not currently widely used. Our hospital has performed RT-3D-TEE examinations on some elderly children. It is hoped that in the near future, RT-3DTEE probes suitable for children will be applied in the examination of pediatric cardiac surgery to provide more intuitive images for the clinic.

\section{Conclusion}

Preoperative TEE can accurately diagnose valvular disease in children and postoperative TEE can find the remaining problems in time and improve the success rate of surgery.

\section{Abbreviations}

Transthoracic echocardiography (TTE)

coronary heart disease (CHD)

ejection fraction (EF)

\section{Declarations}

ETHICS APPROVAL AND CONSENT TO PARTICIPATE 
This retrospective study was approved by the Ethics Committee of Children's Hospital, Zhejiang University School of Medicine(Hangzhou, China). All subjects gave written informed consent in accordance with the Declaration of Helsinki.

\section{CONSENT FOR PUBLICATION}

All parents, guardians or next of kin provided written informed consent for the minors to participate in this study. Written informed consent was obtained from the patient for publication of this case report and any accompanying images. A copy of the written consent is available for review by the Editor of this journal.

\section{AVAILABILITY OF DATA AND MATERIALS}

The analyzed data sets generated during the study are available from the corresponding author on reasonable request.

\section{COMPETING INTERESTS}

The authors declare that they had no competing interests.

\section{FUNDING}

This study was supported by Zhejiang Province Medical and Health Science and Technology Projects (2018KY449).

\section{AUTHOR CONTRIBUTIONS}

JQ performed the examination, collected and analyzed the data, and drafted the manuscript. JY conceived and designed the study, performed the examination. JY helped to collect and analyze the data. $X Y$ helped to perform the study and collected the data. LZ helped to perform the study. All authors read and approved the final manuscript.

\section{ACKNOWLEDGEMENTS}

Not applicable.

\section{References}

1.Puchalski, M. D., et al., Guidelines for Performing a Comprehensive Transesophageal Echocardiographic: Examination in Children and All Patients with Congenital Heart Disease: 
Recommendations from the American Society of Echocardiography. Journal of the American Society of Echocardiography, 2019. 32(2): p. 173-215.

2.Mitchell, C., et al., Guidelines for performing a comprehensive transthoracic echocardiographic examination in adults: recommendations from the American Society of Echocardiography. Journal of the American Society of Echocardiography, 2019. 32(1): p. 1-64.

3.Wang, L., et al., The effect of transesophageal echocardiography in the surgical treatment of tetralogy of Fallot. European review for medical and pharmacological sciences, 2018. 22(7): p. 2084-2087.

4.Guo, Y., et al., Assessment of the mitral valve coaptation zone with 2D and 3D transesophageal echocardiography before and after mitral valve repair. Journal of thoracic disease, 2018. 10(1): p. 283.

5.Tang, X.-J., et al., Intraoperative transesophageal echocardiography in the operation of Ebstein's anomaly: A retrospective study. Chinese medical journal, 2017. 130(13): p. 1540.

6.Yong, M. S., et al., The preoperative evaluation of infective endocarditis via 3-dimensional transesophageal echocardiography. Texas Heart Institute Journal, 2015. 42(4): p. 372-376.

7.Molnar, A., et al., The value of transthoracic and transesophageal echocardiography for the diagnosis of the native aortic infective endocarditis valve complications: a case report and literature review. Medical ultrasonography, 2016. 18(2): p. 253-256.

8.Bai, A.D., et al., Diagnostic accuracy of transthoracic echocardiography for infective endocarditis findings using transesophageal echocardiography as the reference standard: a meta-analysis. Journal of the American Society of Echocardiography, 2017. 30(7): p. 639-646. e8.

9.Pérez-García, C. N., et al., Morphological characterization of vegetation by real-time three-dimensional transesophageal echocardiography in infective endocarditis: Prognostic impact. Echocardiography, 2019. 36(4): p. 742-751.

10. Hosseinsabet, A., Unusual Type of Pulmonary Stenosis Evaluated by 2D and 3D Transesophageal Echocardiography. The Journal of Tehran University Heart Center, 2018. 13(3): p. 153.

\section{Tables}

Table 1. Comparison of preoperative baseline data between the two groups 


\begin{tabular}{|c|c|c|c|c|}
\hline & $\mathrm{TTE} \square \mathrm{N}=64 \square$ & $\mathrm{TEE} \square \mathrm{N}=49 \square$ & $t / Z / X^{2}$ & $P$ \\
\hline Male [N (\%)] & $36(56.25 \%)$ & $28(57.14 \%)$ & 0.009 & 0.924 \\
\hline Age [M (Q25, Q75) month] & $32(11.67,61.5)$ & $37(11.75 \square 66)$ & 0.637 & 0.524 \\
\hline Body mass index (mean $\left.\pm \mathrm{SD}, \mathrm{kg} / \mathrm{m}^{2}\right)$ & $15.72 \pm 1.76$ & $15.48 \pm 2.49$ & 0.607 & 0.545 \\
\hline Heat failure $[N]$ & 0 & 0 & 0.00 & 1.00 \\
\hline Preoperative ejection fraction (mean $\pm \mathrm{SD}, \%$ ) & $63 \pm 3.41$ & $62.83 \pm 3.66$ & 0.244 & 0.808 \\
\hline Heart rate (times/min) & $105.41 \pm 18.40$ & $104 \pm 19.29$ & 0.394 & 0.694 \\
\hline
\end{tabular}

Table 2. Comparison of the compliance rate between TTE and TEE

\begin{tabular}{llll}
\hline METHODS & CASES & THE NUMBER OF CASES CONSISTENT WITH THE & COINCIDENCE RATE WITH \\
& $(\mathrm{N})$ & SURGERY $(\mathrm{N})$ & SURGERY \\
\hline TTE & 64 & 50 & $78 \%$ \\
TEE & 49 & 49 & $100 \%$ \\
\hline
\end{tabular}

Table 3. Comparison of TEE diagnosis and TTE diagnosis in 49 children with TEE 


\begin{tabular}{|c|c|c|c|}
\hline AVSD + Valvular lesion (Figure 1) & 22 & 18 & 22 \\
\hline Tricuspid valvular deformity (Figure 2) & 8 & 8 & 8 \\
\hline $\begin{array}{l}\text { VSD/ASD/PDA + Valvular lesion (Figure } 3 \\
\text { and 4) }\end{array}$ & 8 & 5 & 8 \\
\hline $\begin{array}{l}\text { Aortic arch constriction + PDA + Mitral } \\
\text { stenosis/rupture }\end{array}$ & 1 & 1 & 1 \\
\hline PS + Tricuspid stenosis/rupture & 1 & 1 & 1 \\
\hline $\begin{array}{l}\text { Mitral stenosis with regurgitation (Figure } \\
\text { 5) }\end{array}$ & 2 & 2 & 2 \\
\hline $\begin{array}{l}\text { Anterior mitral valve prolapse }+ \text { posterior } \\
\text { lobe rupture (Figure 6) }\end{array}$ & 1 & 0 & 1 \\
\hline $\begin{array}{l}\text { Aortic valve (bicuspid) malformation } \\
\text { (Figure 7) }\end{array}$ & 2 & 1 & 2 \\
\hline $\begin{array}{l}\text { Moderate regurgitation of aortic-left } \\
\text { ventricular tunnel + aortic valve (Figure 8) }\end{array}$ & 1 & 1 & 1 \\
\hline Infective endocarditis (Figure 9 and 10) & 3 & 2 & 3 \\
\hline
\end{tabular}

AVSD, Atrioventricular septal defect; VSD, Ventricular septal defect; ASD, Atrial septal defect; PDA, Patent ductus arteriosus; PS, Pulmonary stenosis.

Table 4. Cases in which TTE diagnosis was supplemented or corrected by preoperative TEE diagnosis 
NO. TTE diagnosis

1 AVSD (excessive type) + left and right atrioventricular valve moderate regurgitation

2 AVSD (excessive type) + right atrioventricular valve rupture + moderate atrioventricular valve regurgitation

3 AVSD (partial type) + tricuspid valve rupture + mitral midto-severe regurgitation

4 AVSD (complete type) + right atrioventricular valve rupture + left atrioventricular valve mild-moderate regurgitation

$5 \quad$ VSD + mitral and tricuspid valve regurgitation

6 VSD + aortic valve prolapse, moderate insufficiency

$7 \quad$ PDA + anterior mitral valve prolapse with severe regurgitation

8 Left atrial enlargement and moderate mitral regurgitation

$9 \quad$ Aortic valve (tricuspid) and supra-valvular stenosis

10 Posterior mitral valve neoplasm
TEE made supplements or corrections

Multiple ruptures in the left

atrioventricular valve (Figure 1A)

Left atrioventricular valve rupture

Mitral anterior rupture + poor tricuspid development (Figure 1B)

Left atrioventricular valve rupture

Anterior mitral valve prolapse

Congenital rupture of aortic left coronary

valve + non-coronary valve (Figure 3)

Anterior mitral chordae rupture (Figure

4)

Anterior mitral valve prolapse + posterior lobe rupture (Figure 6)

Aortic valve (bicuspid) malformation

(Figure 7)

Posterior mitral valve neoplasm with perforation (Figure 9)

AVSD, Atrioventricular septal defect; VSD, Ventricular septal defect; PDA, Patent ductus arteriosus.

\section{Figures}




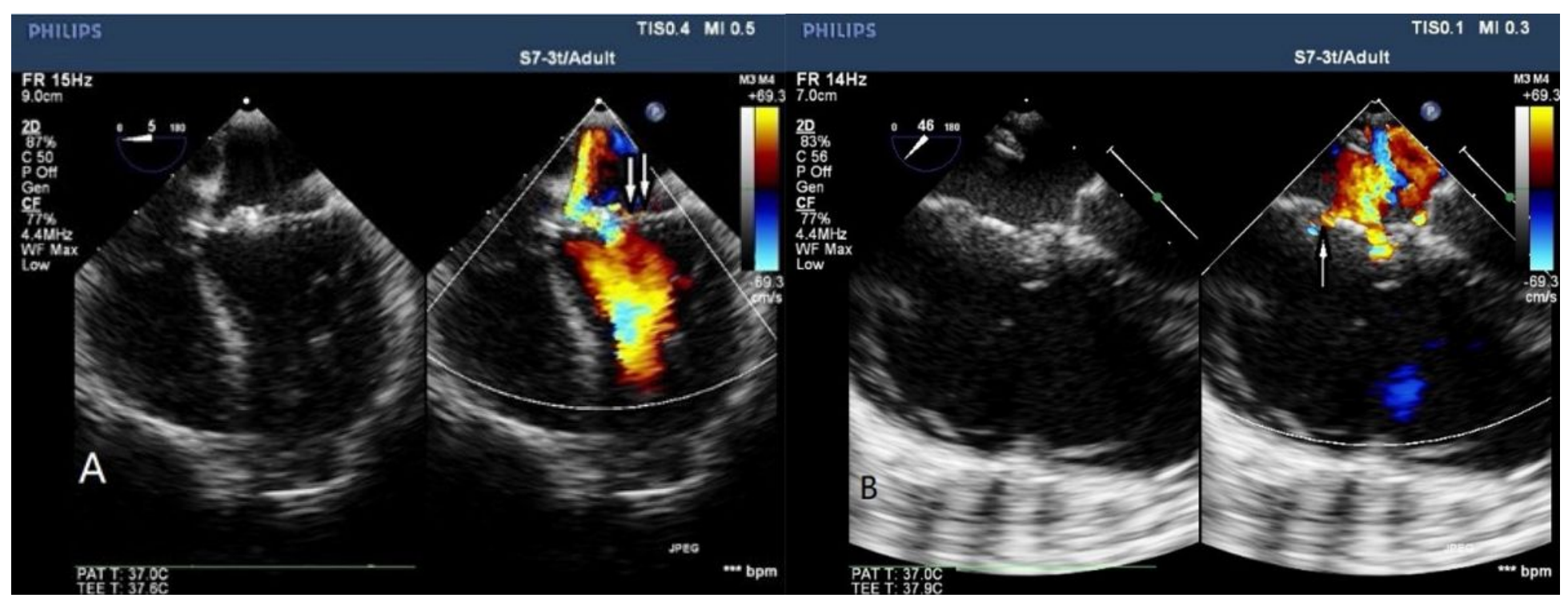

\section{Figure 1}

Atrioventricular septal defect. A: multiple small fissures of the left atrioventricular valve; B: Right atrioventricular valve cleft.

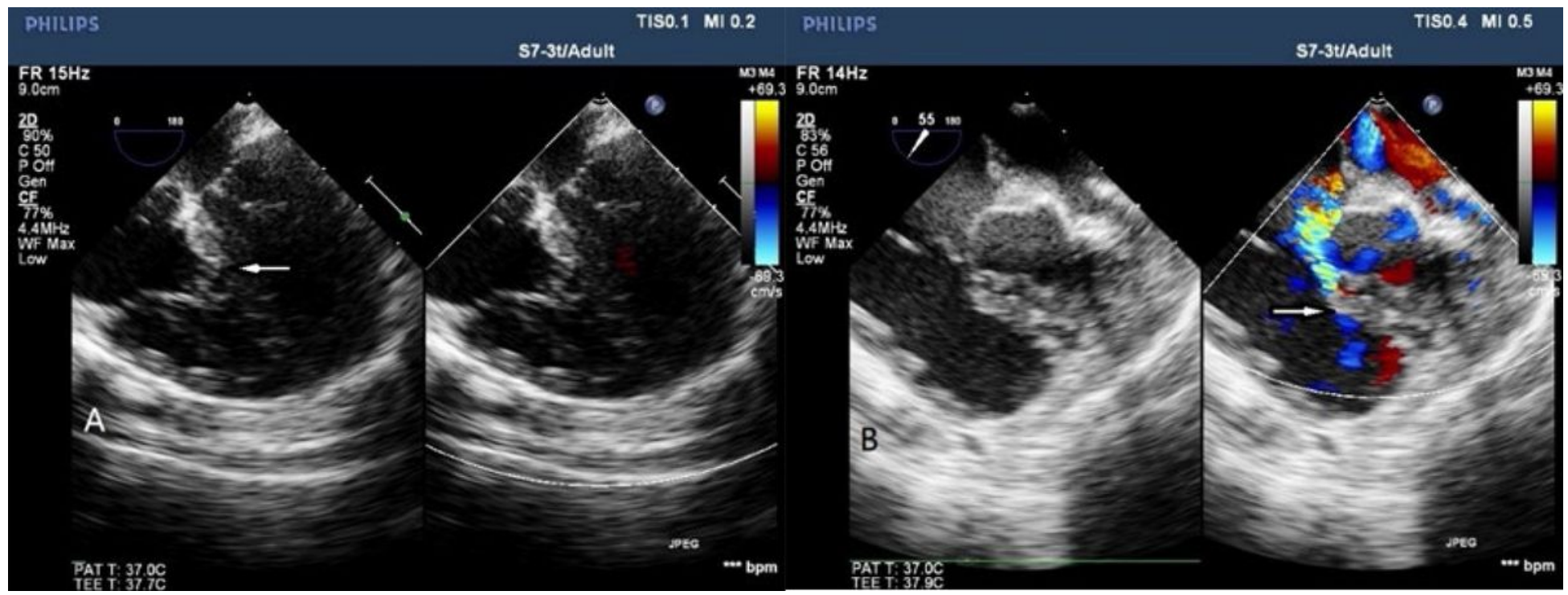

Figure 2

Ebstein's anomaly. A: Cuspis medialis valvulae tricuspidalis moves downward; B: The tricuspid valve is poorly developed and the tricuspid regurgitation is obvious. 


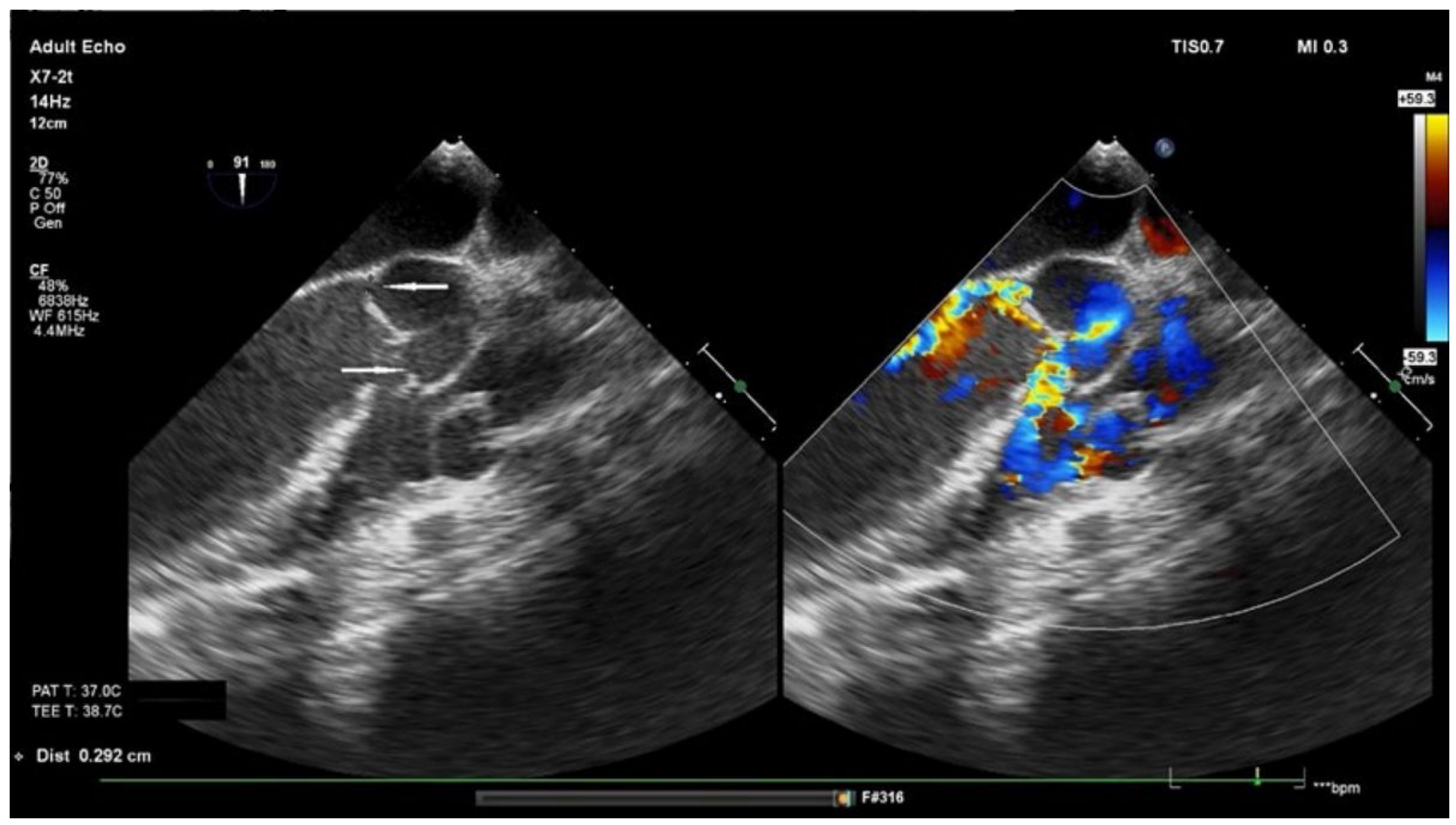

\section{Figure 3}

Ventricular septal defect with aortic valve moderate regurgitation. Preoperative TEE shows ruptures of the aortic left coronary valve and noncoronary cusp.

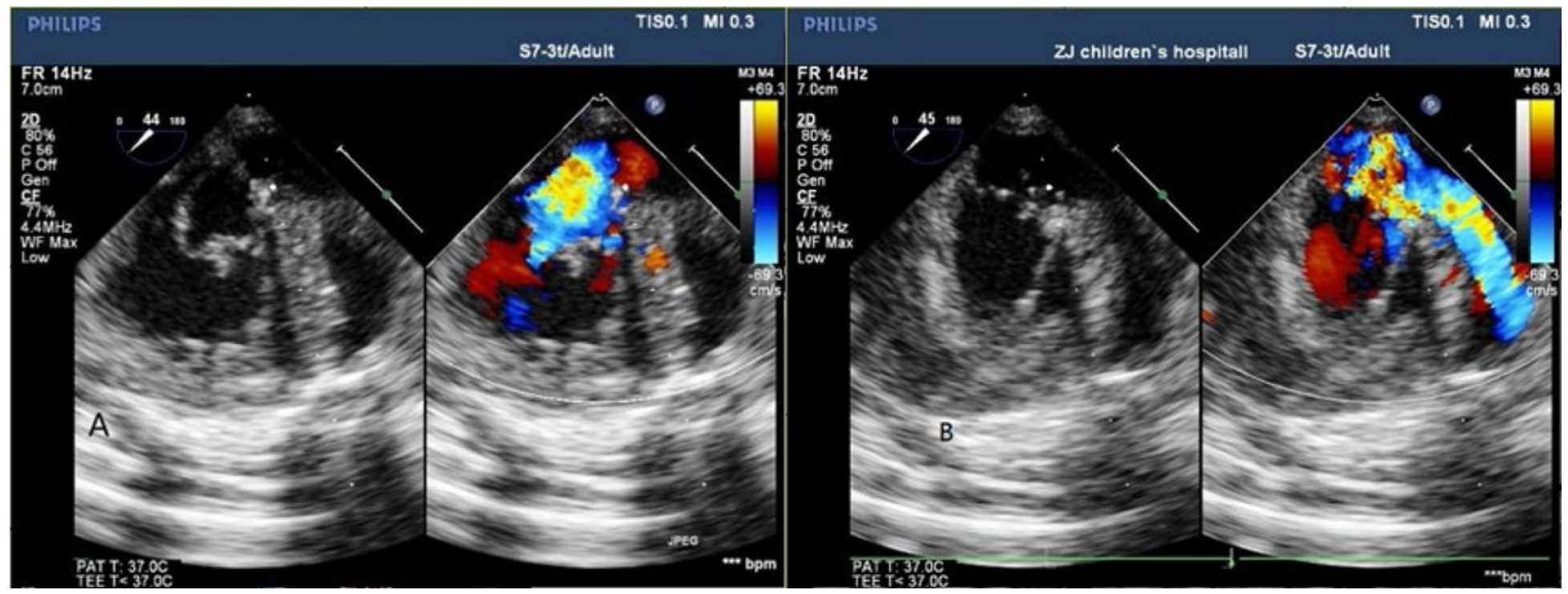

\section{Figure 4}

Patent ductus arteriosus with anterior mitral valve prolapse and severe regurgitation. A: Anterior mitral chordae rupture, where anterior leaflet concaves to the left ventricle as a "door arch" when the mitral valve 
is open; B: Anterior mitral valve is prolapsed into the left atrium when it is closed, leading to severe regurgitation.

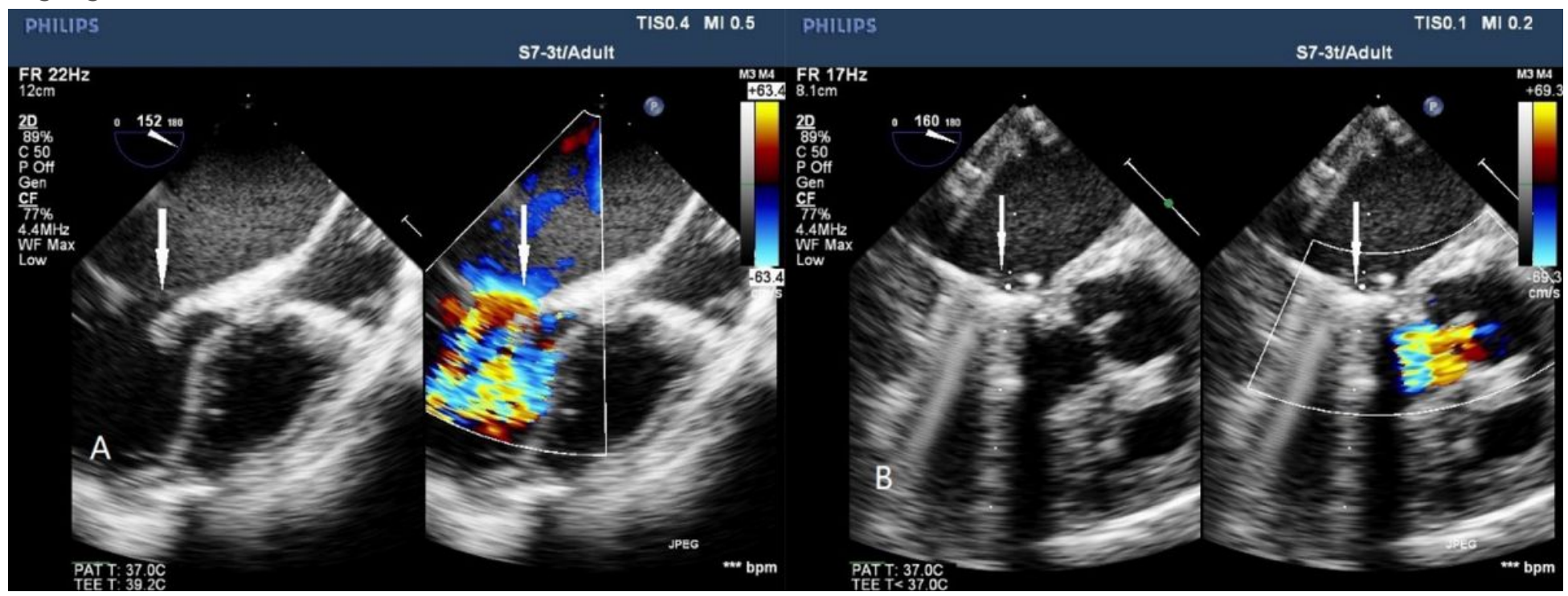

\section{Figure 5}

Mitral stenosis with severe regurgitation. A: Mitral valve echo thickening, enhancement, and open limitation are shown; $\mathrm{B}$ : The mechanical valve activity is well after mitral valve replacement.

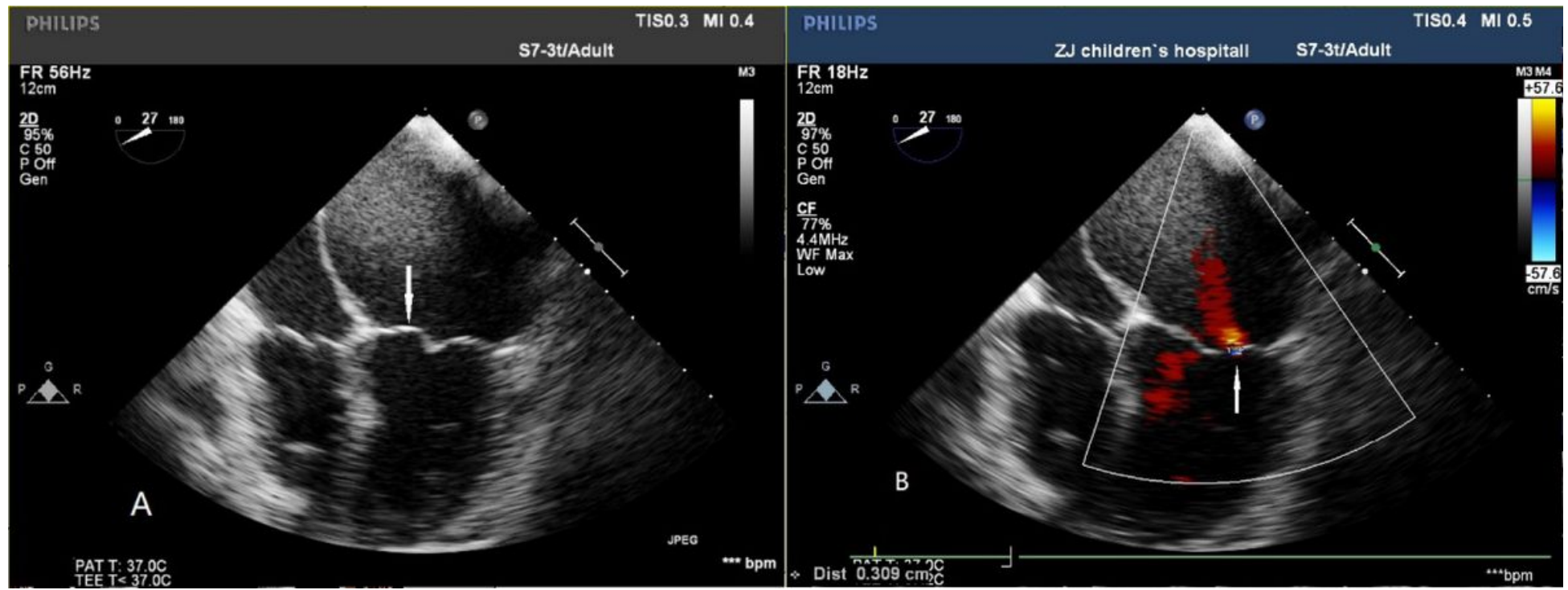

\section{Figure 6}

Left atrial enlargement and moderate mitral regurgitation. A: Anterior mitral valve prolapse; B: Mitral posterior valve cleft. 


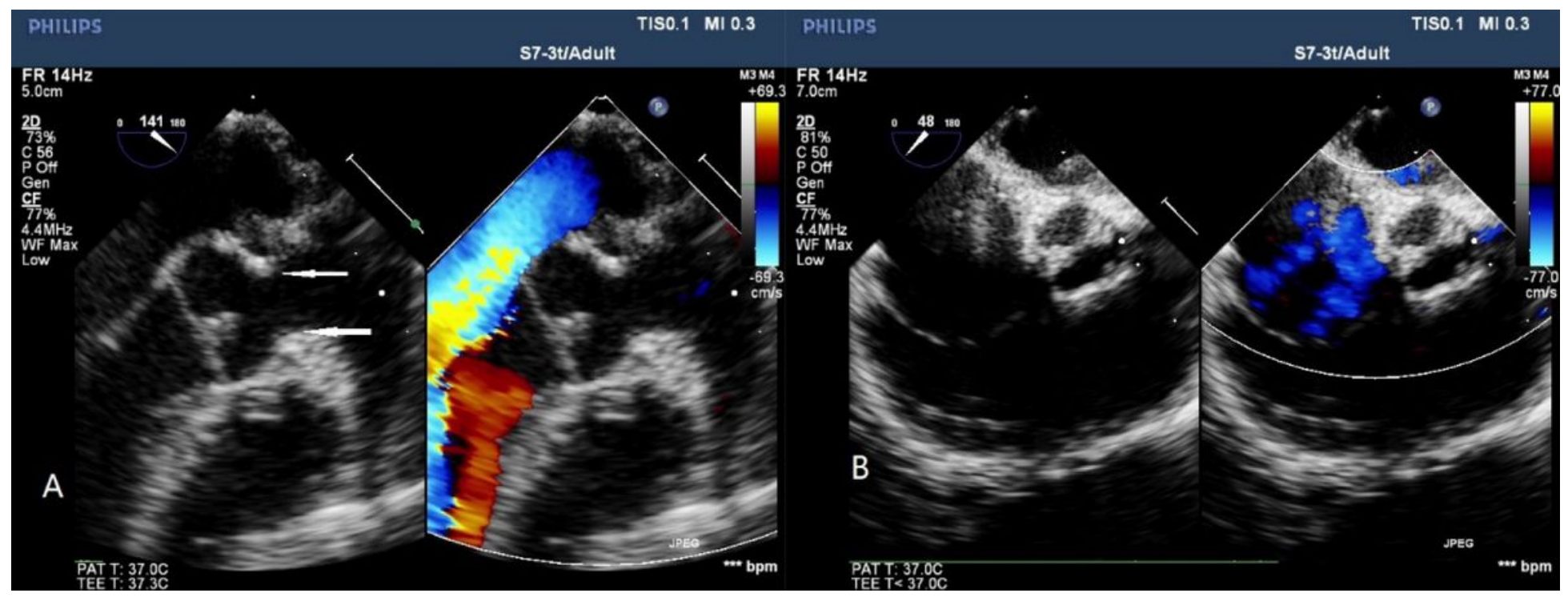

\section{Figure 7}

Aortic valve and supra-valvular stenosis. A: Aortic valve stenosis can be seen on the aortic valve; B: Twolobe malformation of the aortic valve, showing "-" shape when closed.

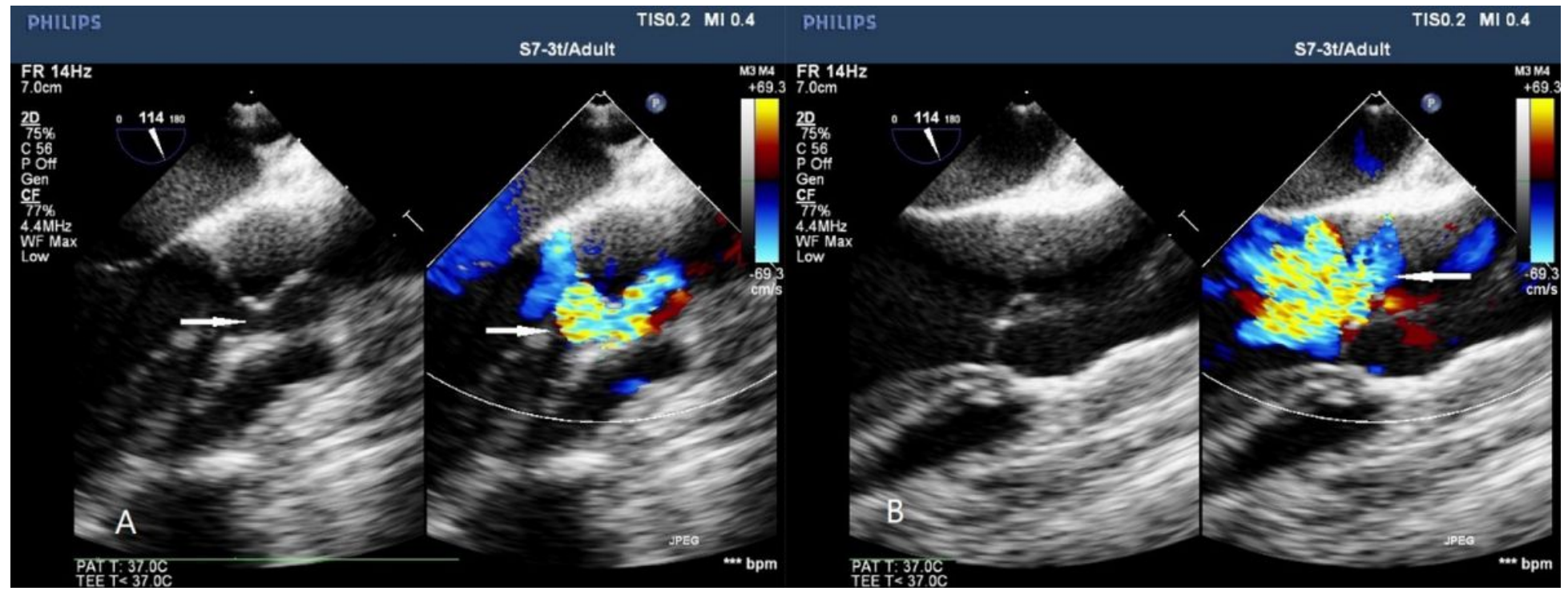

\section{Figure 8}

Aortic-left ventricular canal with aortic valve moderate regurgitation. A: Aorta-left ventricular canal; B: Aortic valve moderate regurgitation. 


\section{PHILIPS}

TISO.1 MI 0.3

\section{ZJ children`s hospitall S7-3t/Adult}

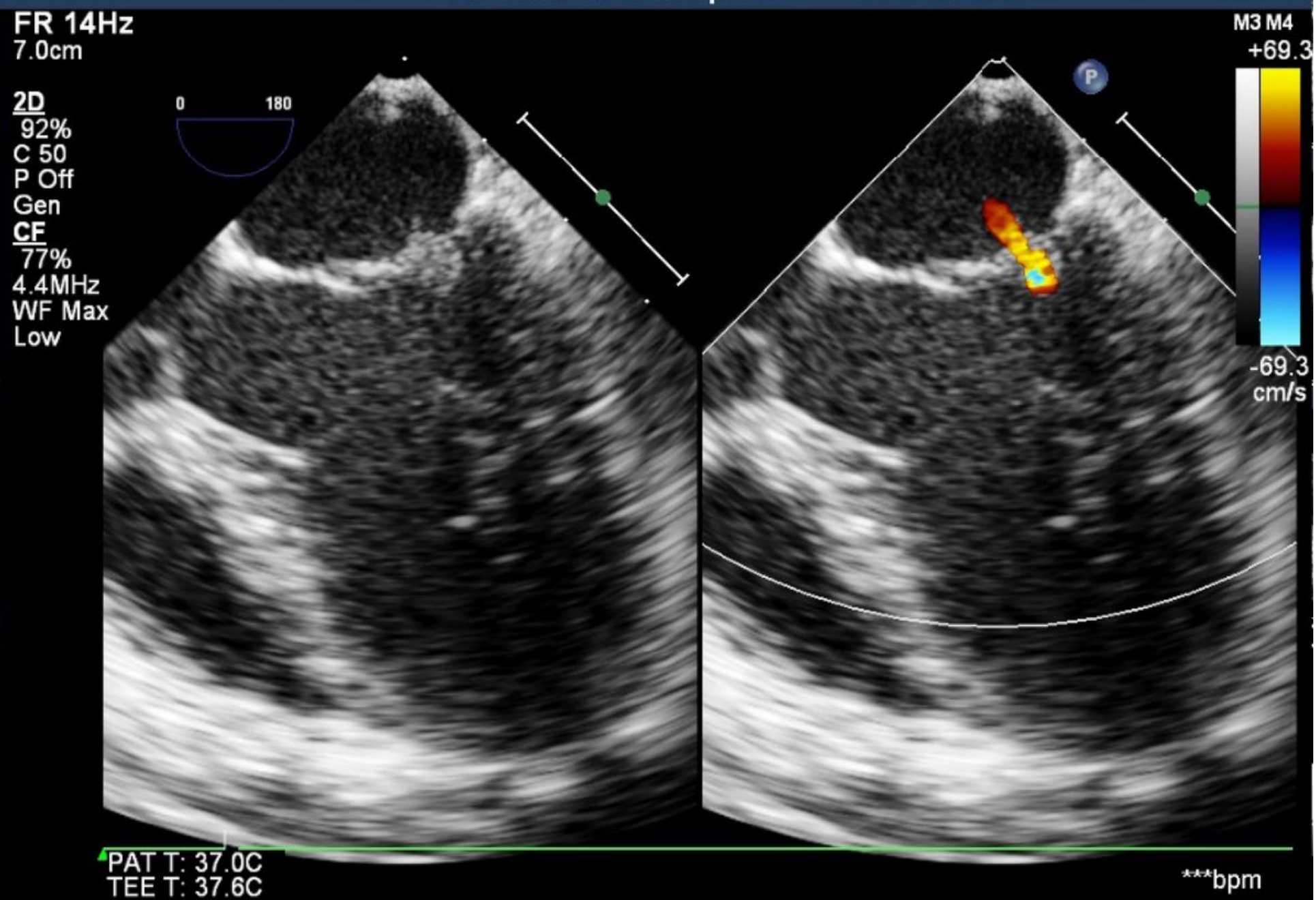

\section{Figure 9}

Infective endocarditis with mitral valve posterior perforation.

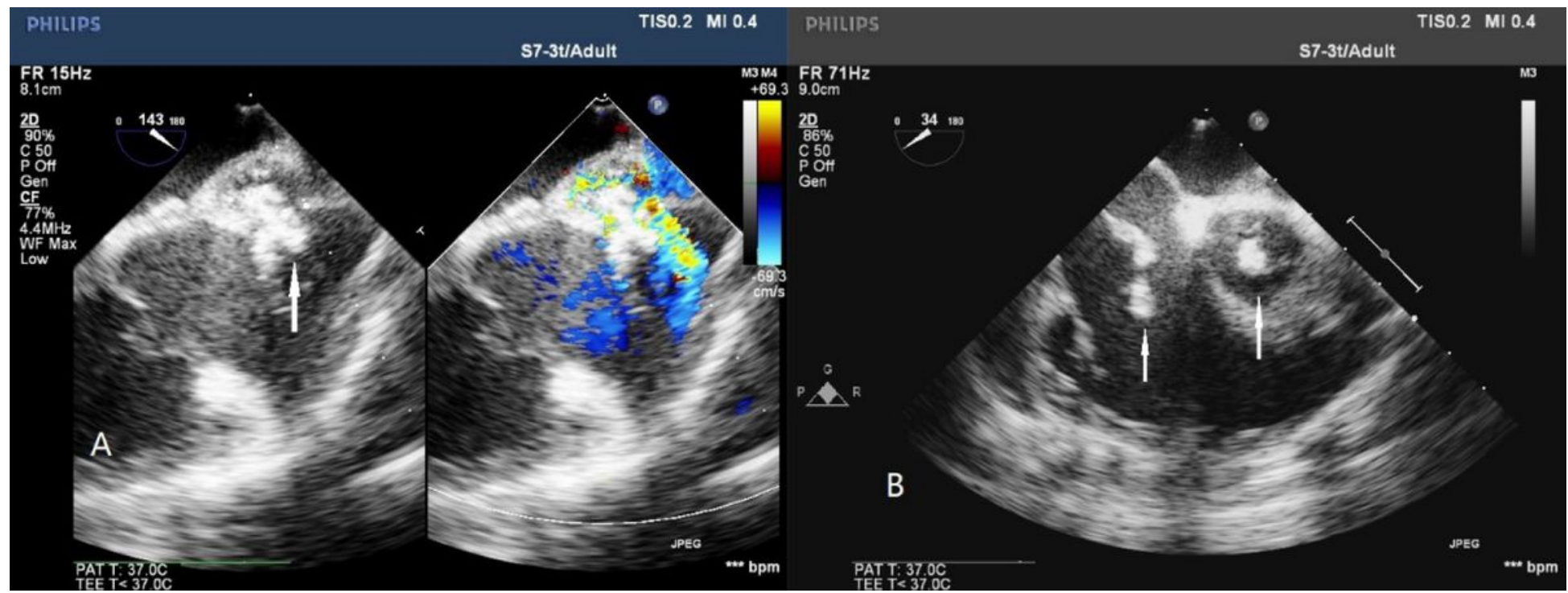


Figure 10

Left coronary artery-right atrial fistula complicated with infective endocarditis. A: The neoplasm is visible in the fistula; $\mathrm{B}$ : The neoplasm continues to the right atrium, and it is also visible in the aortic valve. 